\title{
L'environnement et l'identité écologique dans le roman « Gouverneurs de la rosée » de Jacques Roumain
}

Gina Thésée et Paul R. Carr

\section{OpenEdition}

\section{Journals}

Édition électronique

URL : http://journals.openedition.org/ere/407

DOI : $10.4000 /$ ere. 407

ISSN : 2561-2271

Éditeur

Centr'ERE

Référence électronique

Gina Thésée et Paul R. Carr, «L'environnement et l'identité écologique dans le roman « Gouverneurs de la rosée » de Jacques Roumain », Éducation relative à l'environnement [En ligne], Volume 12 | 2015, mis en ligne le 20 mai 2015, consulté le 10 décembre 2020. URL : http://journals.openedition.org/ere/ 407 ; DOI : https://doi.org/10.4000/ere.407 


\title{
L'environnement et l'identité écologique dans le roman «Gouverneurs de la rosée » de Jacques Roumain
}

\author{
Gina Thésée et Paul R. Carr
}

1 Qui suis-je/sommes-nous? Qui voulons-nous être? Qui pouvons-nous être? Qui savons-nous être? Qui devenons-nous? Dans la réflexion ci-dessus, poétiquement, Jacques Roumain propose la métaphore de l'arbre comme pour mieux aborder l'identité. Moins poétiquement, l'être humain en devenir observe son être, son vouloirêtre, son pouvoir-être et son savoir-être se bousculer, s'affronter dans les rapports de force à l'œuvre dans les processus de construction du soi individuel (Je), du soi collectif (Nous), du soi écologique (Oïkos). Les enjeux identitaires sont depuis toujours au cœur des dynamiques socio-environnementales au Sud comme au Nord, à l'Est comme à l'Ouest, dans les conflits et guerres de genres, de classes, de cultures, de drapeaux, de territoires, de frontières, d'ethnies, de races, de religions ou d'idéologies. Ces enjeux suggèrent que l'on ne peut ni dissocier l'identité, qui est ouverture à soi, de son « alterego ", l'altérité, qui est ouverture au Nous et à l'Autre, ni faire fi des tensions sousjacentes inévitables, voire, nécessaires entre ces deux pôles résolument solidaires. Derrière ces enjeux identitaires sont à l'œuvre d'âpres débats de nature éthique, politique et critique, mais aussi philosophique et spirituelle. Ainsi, les luttes pour la paix, l'équité, la justice, la démocratie, la santé, l'éducation, l'inclusion et l'environnement posent avec acuité la question des personnes, dans leurs droits fondamentaux, individuels et collectifs, mais aussi dans leurs rapports à soi, à l'Autre, au savoir et au monde. Comment les tensions identitaires des corps physique, social et environnemental peuvent-elles se résoudre dans la construction d'une identité écologique?

2 En analysant le roman "Gouverneurs de la rosée» de l'écrivain haïtien Jacques Roumain, nous cherchons à comprendre comment le rapport à l'environnement 
contribue à façonner l'identité individuelle, sociale et écologique, et réciproquement, comment ces identités contribuent à façonner le rapport à l'environnement. C'est par l'analyse de l'identité/altérité du personnage Manuel, dans ses dimensions sociopolitique et écologique, que nous recherchons les réponses. Comme le dit Roumanes (1993), depuis le triomphe de la linguistique comme science dans les années 60-70, la psychanalyse et la psychologie ont pu se doter d'un objet d'étude qui leur faisait défaut: le langage. C'est donc à travers le langage Romanesque de l'auteur Jacques Roumain, que nous mettons en évidence dans le texte, que nous allons tenter de saisir l'identité dans ses dimensions socio-politique et écologique. Nous sommes conscients que notre démarche peut sembler atypique dans le cadre d'une revue académique en éducation, mais nous souhaitons justement mettre en scène les mots, les images, les métaphores, les tournures de phrases et l'imaginaire de l'auteur qui tissent le caractère écologique et éducatif du roman. Nous souhaitons montrer la portée écologique du discours pour ensuite en tirer ce qui nous parait comme une proposition d'éducation à l'identité écologique, et ce, vingt ans avant la naissance de l'écologie.

Nous menons cette analyse en présentant: dans la première section, une problématique sociopolitique et environnementale d'Haïti; dans la deuxième, l'engagement sociopolitique de l'auteur Jacques Roumain; dans la troisième, l'analyse du roman "Gouverneurs de la rosée "; dans la quatrième, l'analyse de l'environnement comme personnage secondaire du roman; dans la cinquième, l'analyse de l'identité écologique du personnage Manuel; dans la sixième, la portée du roman dans le contexte actuel d'Haïti; et dans la dernière section, la mise en évidence d'une proposition d'éducation à l'environnement et à l'identité écologique.

\section{Problématique sociopolitique et environnementale du contexte haïtien}

Quelques années après le séisme qui a dévasté Haïti le 12 janvier 2010, la problématique socio-environnementale se pose toujours comme une douloureuse réalité quotidienne à la fois évidente et ignorée, qui n'occupe pas une très grande place dans les discussions et débats. Reconstruire quoi? Reconstruire comment? Et surtout, comment reconstruire autrement? En ce sens, certains proposent non pas la reconstruction, mais bien la «refondation» d'Haïti (GRAHN, 2010). Il s'agit alors de repenser et de poser de nouvelles bases en vue de la fondation d'une Haïti nouvelle. L'effet dévastateur du séisme ne peut s'expliquer par sa seule intensité, établie à 7,4 sur l'échelle de Richter, puisque de nombreux séismes de même intensité n'ont pas provoqué une telle désolation : près de 300000 victimes et d'innombrables blessés graves physiquement et psychologiquement, sans parler de la destruction d'un grand nombre de bâtiments résidentiels et institutionnels. Selon Bourjolly (2010), l'effet le plus dévastateur de ce séisme est l'effondrement des capacités d'Haïti. Bien avant le séisme, et même depuis sa naissance il y a deux siècles, Haïti était une terre livrée aux multiples dynamiques de violences institutionnalisées : violences de l'esclavage, violences coloniales, violences envers les femmes, violences envers les enfants, violences envers les phénotypes, violences envers les citoyens, violences envers certains groups sociaux, violences envers la ruralité, violences envers l'environnement-culture, violences envers l'environnement-nature. La résultante de toutes ces violences est une société qui s'est façonnée, au fil de ses deux siècles d'existence, par les inégalités et les injustices 
sociales, la pauvreté criante, la domination des femmes, l'exclusion de certains groupes sociaux, une suite presque ininterrompue de dictatures, l'émigration massive, une dépendance totale envers l'extérieur, l'érosion des sols, les vulnérabilités socioenvironnementales (Carr, Pluim et Thésée, 2014). Les droits humains fondamentaux comme l'eau potable, l'alimentation, l'emploi, le logement, la santé, l'éducation et la parole sont refusés à la très grande majorité de la population haïtienne sans soulever d'indignation. Le projet de pays initié au moment de la guerre contre l'esclavage et le colonialisme s'est enlisé dans le prolongement et la reproduction de ces mêmes dynamiques esclavagistes et coloniales. L'indépendance d'Haïti a permis de rompre les chaînes aux pieds des esclaves, mais elle n'a pas encore permis de rompre les chaînes dans l'esprit de leurs descendants qui maintiennent intacts les rapports de pouvoir esclavagistes et coloniaux.

Dans son roman « Gouverneurs de la rosée », Jacques Roumain recourt à la poétique du langage vernaculaire haïtien pour tisser les liens inextricables entre l'environnementnature et l'environnement-culture du pays, entre l'écologie et le social d'un petit village, entre l'identité et l'altérité des villageois, entre le cœur et la raison d'un jeune homme amoureux. Ce faisant, il invite à "refonder " les rapports à soi, à l'Autre, au savoir et à l'environnement, non seulement au plan local, en Haïti, mais aussi au plan global, car son roman écologique a bel et bien une portée de mondialité au sens où l'entend Édouard Glissant.

\section{Réflexions sur l'identité de l'auteur Jacques Roumain à la lumière de son engagement socio-politique, scientifique et littéraire}

Chaque pays a son Roumain, c'est-à-dire un écrivain qui résume en quelque sorte les rêves, les élans et les échecs de sa société... C'est plutôt quelqu'un qui n'hésite pas à assener ses quatre vérités à ses compatriotes. Pour le Québec, je pense à Miron. Pour Haïti, c'est Roumain. (Laferrière cité dans Roumain, 2007, quatrième de couverture)

6 À travers la quête identitaire du personnage central du roman «Gouverneurs de la rosée ", celle de l'auteur transparaît nettement: Roumain, le patriote outré face au massacre des Haïtiens ordonné par l'eugéniste président dominicain Trujillo ; Roumain, le révolté face aux forces d'occupation américaine en Haïti de 1915 à 1934 ; Roumain, l'indigné face à la coopération du gouvernement avec les forces d'occupation; Roumain, le camarade solidaire dans les luttes des classes prolétaires; Roumain, le citoyen engagé co-fondateur du premier Parti communiste haïtien; Roumain, l'indigéniste qui promeut la culture première de son pays ; Roumain l'exilé ; Roumain, le voyageur qui, en anglais, en allemand, en espagnol ou en français étudie les sociétés dans lesquelles il séjourne comme pour mieux en faire profiter la sienne à son retour ; Roumain, le scientifique pluridisciplinaire qui recourt à l'ethnologie, l'anthropologie, la paléontologie et l'archéologie pour mieux comprendre sa société et sa terre, celles d'hier et d'aujourd'hui; Roumain, l'enseignant et passeur qui veut transmettre les savoirs requis à sa terre natale; Roumain, le prisonnier qui, inlassablement, dénonce et combat les dérives sociales armé de ses seuls mots. Jacques Roumain ne se dérobe pas; il assume pleinement sa colère, l'exprime et la confie à Manuel son personnage à qui il fait dire : La rage te fait serrer les mâchoires et boucler ta ceinture plus près de la peau de ton 
ventre quand tu as faim. La rage, c'est une grande force... La rage, c'était son droit et sa justice (Roumain, 2007, p. 19).

7 Précédant Aimé Césaire, Léopold Senghor, Frantz Fanon, Albert Memmi, Malcom X, Martin Luther King et combien d'autres, Jacques Roumain fait figure d'un guerrier épistémologique pionnier dans sa résistance face aux projets d'occupation, de domination et d'oppression, qui prend des formes diverses telles l'action sociale, les prises de position publiques, le journalisme politique, la traduction d'œuvres engagées, la recherche scientifique, les conférences et la littérature. Son action sociale donne lieu notamment à la co-fondation de la Revue Indigène en 1927, à la présidence de la Ligue de la Jeunesse Patriote Haïtienne en 1928, à la rédaction en chef du journal «Le Petit Impartial » en 1928, à la fondation du Parti Communiste Haïtien en 1934, à la fondation du Bureau d'Ethnologie d'Haïti en 1942 et à la fondation de l'Institut international d'études afro-américaines à Mexico en 1942. Ses prises de position politiques sont exprimées dans de nombreux essais publiés dans diverses revues dont «Analyse schématique 1932-1934 », «Les griefs de l'homme noir ». Comme journaliste politique, il dirige le journal Le Petit Impartial défavorable au gouvernement de l'époque et compte à son actif de nombreux articles qui dénoncent avec véhémence des faits sociaux d'Haïti et de l'étranger.

Qui était Jacques Roumain? Selon l'auteur lui-même, résolument un Haïtien qui, de retour en Haïti à l'âge de vingt ans après quelques années d'études en Europe, exprime ainsi son soulagement : il se reconnaissait enfin... écoutant fondre en lui la glace amassée en Europe, disparaître de son cœur ce qu'il nommait avec amertume «le grand silence blanc »... Maintenant, il était parmi ses frères et son peuple (Roumain dans La Proie et l'ombre, p. 59, cité par Hoffmann, 2003). Paradoxalement, l'haïtianité de Roumain s'est d'abord tissée dans un milieu socioéconomique privilégié, son père étant un grand propriétaire terrien et son grand-père maternel ayant été président de la République lorsqu'il avait cinq ans. De plus, compte tenu du contexte "socio-épidermique" d'Haïti, Jacques Roumain fait partie de l'élite mulâtre, ce qui lui confère de facto des privilèges de classe, mais qu'il a osé remettre en question. Par ailleurs, ses séjours prolongés en Suisse, en Espagne, en Belgique, en France, aux États-Unis, à Cuba et au Mexique associés au fait qu'il maîtrise plusieurs langues dont le créole, le français, l'espagnol, l'allemand et l'anglais, font de lui un citoyen du monde ouvert à l'Autre et bien imprégné des cultures qu'il traverse. Cependant, ses racines identitaires plongent profondément dans le sol d'Haïti avec une passion doublée d'une vigilance critique aiguisée.

9 L'activisme sociopolitique de Roumain n'est pas fortuit, il s'y est longuement préparé et, même peu avant sa mort, il s'estimait non suffisamment prêt, il voulait continuer à apprendre, à essayer de comprendre son pays : Il me disait en 1944 avoir senti le besoin d'une préparation plus profonde, qui le mette à même de mieux comprendre la société haïtienne (Bastien, 1954, cité par Hoffmann, 2003). C'est un activiste réfléchi qui s'appuie sur des savoirs: Je m'applique à me préparer... Fils de grands propriétaires terriens, j'ai renié mes origines bourgeoises. J'ai beaucoup vécu avec les paysans. Je connais leur vie, leur mentalité, leur religion, ce mélange étonnant de catholicisme et de vaudou (Roumain dans une Lettre à Tristan Rémy, cité par Fombrun, 2007). Il est sans complaisance : Je ne considère pas le prolétariat paysan comme un trésor sentimental. Le paysan est notre seul producteur et il ne produit que pour être exploité de la manière la plus effroyable, par une minorité politicienne qui s'intitule l'Élite. Tous mes écrits ont combattu cette prétendue élite... (Idem). 


\section{Gouverneurs de la rosée : un roman-essai sur la solidarité socio-environnementale}

Mais ce n'est pas seulement la manière de l'écrivain qui, dans Gouverneurs de la rosée, est à son zénith. C'est aussi le talent de l'ethnologue qui y atteint son acmé. [...] observations ethnographiques et ses réflexions ethnologiques, dans leur substance, et sous une forme adéquate, passent dans son roman et l'enrichissent de cette matière qui en fait un admirable document sociologique.

(Souffrant, 1976, dans Hoffmann, 2003)

Le roman Gouverneurs de la rosée de Jacques Roumain, qui constitue son testament paru à titre posthume en 1944, est certainement l'œuvre littéraire haïtienne la plus connue et la plus célébrée dans le monde. Traduit dans une vingtaine de langues, réédité plusieurs fois, transposé au théâtre, analysé dans des thèses et mémoires, et étudié dans des programmes scolaires, ce roman de la paysannerie se fait l'écho de préoccupations sociales, politiques et environnementales avec une sensibilité poétique qui prend sa source dans le créole vernaculaire haïtien, comme l'illustre le titre Gouverneurs de la rosée traduction de "Mèt lawouze », tout en se permettant de franches libertés linguistiques avec le français comme « une saison malédictionnée ». Ce roman est aussi le reflet d'une observation ethnographique fine, le souffle d'une analyse anthropologique rigoureuse, l'écho universel d'un essai sociologique haïtien (Hoffman, 2003).

11 La misère s'est abattue sur les villageois de Fonds-Rouge qui constatent, avec un sentiment d'impuissance et de résignation, l'état de désolation de leur environnement depuis que la sécheresse s'y est installée : Nous mourrons tous... et elle plonge sa main dans la poussière; la vieille Délira Délivrance dit: nous mourrons tous: les bêtes, les plantes, les chrétiens vivants, ô Jésus Marie Sainte Vierge ; et la poussière coule entre ses doigts (Roumain, 2007, p. 5). Bienaimé, son mari, pense : On était déjà mort dans cette poussière, cette cendre tiède qui recouvrait ce qui autrefois avait été la vie... (p. 13). Pendant que la femme prie Dieu et tous les saints de les délivrer de ce grand malheur, l'homme peste contre ce même Dieu pour leur avoir envoyé ce malheur : Le Seigneur, c'est le créateur du ciel et de la terre, pas vrai ? [...] Eh bien, la terre est dans la douleur, la terre est dans la misère, alors, le Seigneur c'est le créateur de la douleur, c'est le créateur de la misère. (p. 6). Les deux conjoints, d'un élan commun, remettent la cause et la solution de leurs problèmes à l'extérieur de leur champ de possibilités, entre les mains d'une divinité qu'ils prient ou invectivent, tous deux assujettis à une même logique d'abdication de leur propre pouvoir d'action et à la résignation comme seul mécanisme de résilience. Délira se sent abandonnée par ces divinités sourdes et aveugles d'Afrique qui ne l'avaient pas entendue...? (Roumain, 2007, p. 15).

12 Une autre misère tout aussi désolante a frappé Fonds-Rouge, C'était la haine et son ruminement amer du passé sanglant, son intransigeance fratricide (Roumain, 2007, p. 41). Cette misère-là assèche les cœurs provoquant l'érosion des relations interpersonnelles, du tissu social, de la vie communautaire et de la solidarité pour une histoire de partage de terres ayant semé la mort (p. 41). Ils appartiennent désormais au passé faisant place à l'indifférence et à l'isolement. Érosion environnementale et érosion sociale sont le reflet l'une de l'autre, s'entretiennent mutuellement, les deux calamités appartenant à une même dynamique d'absence de soins, care, de compassion, de soin de soi, de soin de l'Autre et de soin de l'environnement. La réaction des villageois devant cette calamité 
sociale est semblable à leur réaction devant la sécheresse : un sentiment d'impuissance et de la résignation.

Une autre misère, affective celle-là, vécue depuis par Délira et Bienaimé est sans contredit l'absence de Manuel leur fils unique parti depuis quinze ans pour couper la canne à sucre à Cuba. Pour celui qui est parti, comme pour ceux qui sont restés, la migration a eu des effets désolants qui défont les liens d'attachement, provoquent une érosion familiale qui vient s'ajouter aux effets des érosions environnementale et sociale. L'émigration frappera bientôt le village et sera vue comme un phénomène tragique inéluctable. Cette représentation de l'émigration comme une saignée subie par le village semble annoncer un phénomène qui se produira réellement à l'échelle nationale une vingtaine d'années plus tard, l'exode massif des Haïtiens fuyant la dictature et la pauvreté vers les pays étrangers. À Fonds-Rouge, c'est le retour de Manuel qui dénouera le triple nœud de leur misère: l'érosion environnementale, sociale et familiale.

Manuel, de retour pour le restant de sa vie sur sa terre natale assoiffée, apporte avec lui un souffle rafraîchissant venu de Cuba, là où l'eau coule d'un bout à l'autre des plantations (Roumain, 2007, p. 29). Toutefois, Manuel ne s'y méprend pas, cette reconnaissance des attributs de la terre cubaine ne l'empêche pas d'être déçu de la société cubaine et d'exprimer une critique sociale sévère qui n'est pas sans rappeler la situation actuelle des Haïtiens dans les bateys en République dominicaine où l'identité haïtienne est toujours en péril : " Haitiano maldito, negro de mierda » hurlaient les gardes. Les coups ne faisaient même plus mal. ; Matar a un Haitiano o a un perrro : tuer un Haittien ou un chien, c'est la même chose disent les hommes de la police rurale : de vraies bêtes féroces (p. 23, 30).

\section{L'environnement : un personnage secondaire important}

Il n'est pas étonnant que l'environnement ne soit pas mentionné ou que la dimension écologique du roman soit passée sous silence. Aujourd'hui encore, même avec le mouvement écologique, et près de soixante-dix ans après sa parution, cet aspect du roman est peu mis en évidence et sa pédagogie environnementale n'est pas mise à profit pour concevoir ce qui pourrait constituer une éducation à l'environnement dans le contexte socio-environnemental d'Haïti et d'ailleurs. Pourtant, le roman met en scène l'environnement comme un important personnage secondaire, complexe et systémique, dont les multiples dimensions font écho à la typologie des représentations de l'environnement décrites par Sauvé (2001, p. 298) :

«l'environnement - nature à apprécier, respecter et préserver ; l'environnement ressource à gérer; l'environnement - problème à résoudre; l'environnement système à comprendre pour décider ; l'environnement - milieu de vie à connaître et à aménager ; l'environnement - biosphère où vivre ensemble et à long terme ; l'environnement - projet communautaire où s'engager ".

L'environnement - nature (à apprécier, respecter, préserver) est décrit en termes de désolation dans le regard de Manuel sur son village : la terre est dans la douleur, la terre est dans la misère; Le ciel n'a pas une fissure. Ce n'est qu'une plaque de tôle brûlante; les érosions ont mis à nu de longues coulées de roches: elles ont saigné la terre jusqu'à l'os (Roumain, 2007, p.6). C'est dans l'évocation d'un passé lointain que l'on trouve des représentations positives de l'environnement-nature : On entrait dans l'herbe de Guinée! Les pieds nus dans 
la rosée... (Roumain, 2007, p. 7); dans les coques dorées des assorossis s'épanouissait une pulpe rouge comme un velours de muqueuses (p. 8); Le chant emplissait le matin inondé de soleil. Le vent l'emporterait au-delà des collines; Le chant obstiné des coqs alternait d'un jardin à l'autre (p. 9) ; Le chant prendrait le chemin des roseaux, le long du canal [...] il remonterait jusqu'à la source tapie au creux de l'aisselle du morne, dans la lourde senteur des fougères et des malangas macérés dans l'ombrage et le suintement secret de l'eau (p. 10).

L'environnement - ressource (à gérer) est surtout désigné en des termes associés à la gestion des récoltes du passé, à l'incapacité de gérer les maigres récoltes du présent : $i l s$ avaient incendié le bois pour faire des jardins de vivres... (Roumain, 2007, p. 17) ; Le café, le coton, le riz, la canne, le cacao, le maïs, les bananes, les vivres et tous les fruits, si ce n'est pas nous, qui les fera pousser (p. 51); Les habitants tiraient des vaches un peu de lait de méchante qualité (p. 53). En plus des vivres alimentaires, ce sont le bois et le charbon de bois qui constituent pour eux la principale source de revenus : On a éclairci pour le bois-neuf, on a coupé pour la charpente et le faittage des cases, on a refait les entourages des jardins... (p. 37). Cependant, malgré leur dur labeur, les ressources suffisent à peine à les faire vivre (p. 19). L'arbre appelé bayahonde, et dont le nom scientifique est Prosopis Juliflora, est souvent présent dans la description des lieux du village : Aussitôt que le soleil touchait le bois, assez pour éclairer à travers les bayahondes les sentiers entrecroisés, Manuel partait. Il abattait les arbres, dressait dans la clairière la meule sous laquelle le bois brûlerait à feu lent (p. 49). L'omniprésence des bayahondes souligne l'importance de cet arbre dans la fabrication du charbon de bois. Des agronomes haïtiens soutiennent que l'abattage de cet arbre présente moins d'impacts négatifs sur l'environnement que la coupe des arbres fruitiers (Jeanty, 2012).

L'environnement - problème (à résoudre) est abondamment évoqué, mais plutôt en des termes d'impuissance et de résignation par les villageois : La terre est toute nue et sans protection. [...] Mais la terre est comme une bonne femme, à force de la maltraiter, elle se révolte... (Roumain, 2007, p. 25); il n'y a plus rien: l'eau est tarie depuis les entrailles du morne, c'est pas la peine de chercher plus loin, parce que c'est inutile. (p. 37); les érosions ont mis à nu de longues coulées de roches: elles ont saigné la terre jusqu'à l'os. (p. 6). Le manque d'eau est la conséquence environnementale de l'incurie des villageois conséquente à leur pauvreté : l'érosion des sols à la suite du déboisement effréné pour produire du charbon de bois.

L'environnement - système (à comprendre pour décider) révèle l'incompréhension des villageois des liens de cause à effet entre leurs actions et les conséquences environnementales qu'ils subissent. Manuel s'évertue à le leur expliquer pour qu'ils comprennent l'urgence de rétablir l'équilibre rompu: Ce sont les racines qui font amitié avec la terre et la retiennent: ce sont les manguiers, les bois de chênes, les acajous qui donnent les eaux des pluies pour sa grande soif et leur ombrage contre la chaleur de midi (Roumain, 2007, p. 25). Sa compréhension de l'environnement-système lui a permis de trouver l'eau et même d'en apprécier la quantité : c'est une eau conséquente. Faut voir l'endroit: c'est une grande terrasse de terre blanche comme la craie; ça boit l'eau facilement cette qualité de terre, mais l'eau a dû trouver plus loin du dur, du résistant, alors elle a gonflé. Sûr que dans quelques années, elle aurait crevé toute seule (p. 92).

L'environnement - milieu de vie (à connaître, à aménager) fait appel aux sens, aux sentiments et à la sensualité des corps comme pour mieux développer son " être-aumonde» puisque «Le monde se donne d'abord sous la forme du sensible. Notre condition est corporelle. Il n'est rien dans l'esprit qui n'ait séjourné dans les sens » (Le 
Breton, 2006, cité par Cottereau, 2012, p. 19). Pour mieux habiter le lieu et y développer l'appartenance, c'est d'abord le corps qui « absorbe ou refuse, nous relie ou nous sépare [...] il est au cœur de notre saisie du monde » (Cottereau, 2012, p. 17). Dans le roman, on voit que c'est par les sens que les villageois habitent et s'approprient leur milieu de vie : dès le détour du chemin, une odeur venait à leur rencontre, les saluait positivement, les enveloppait, les pénétrait, leur ouvrait dans l'estomac le creux agréable du grand goût (Roumain, 2007, p. 12).

21 L'environnement - biosphère, jusqu'au retour de Manuel, est circonscrit à l'intérieur des limites du village et ne va pas au-delà des marchés où les villageois de Fonds-Rouge se rendent pour vendre leurs provisions. Mais la misère va les forcer à envisager un ailleurs meilleur et la biosphère prend alors le nom des lieux d'émigration, car : Qu'estce que tu veux, cette sécheresse, c'est décourageant et il y en a qui ne se résignent pas à périr ; ils préfèrent quitter la terre des anciens pour aller chercher la vie en pays étranger (Roumain, 2007, p. 75). Le retour de Manuel amplifie leur perspective en leur apportant des informations sur l'ailleurs: Oui, mais à Cuba, il y a plus de richesse, on vit plus à l'aise. Icitte, il faut se gourmer dur avec l'existence et à quoi ça sert? On n'a même pas de quoi remplir son ventre et on est sans droit contre la malfaisance des autorités (p. 50).

L'environnement - projet communautaire (où s'engager) appartient au passé lointain du village : Une circulation rythmique s'établissait entre le cœur battant du tambour et les mouvements des hommes: le rythme était comme un flux puissant qui les pénétrait jusqu'au profond de leurs artères et nourrissait leurs muscles d'une vigueur renouvelée (Roumain, 2007, p. 9). Dans le présent, il est contrecarré par les conflits et la haine que se vouent les villageois, les liens de solidarité étant rompus : Chacun garde sa rancune et fourbit sa colère. Il y a nous et il y a les autres. Et entre les deux: le sang. On ne peut traverser le sang (p. 41). Manuel parvient à instiller au village un esprit de démocratie participative, dialogique, solidaire : c'est la plus grande chose au monde que tous les hommes sont frères, qu'ils ont le même poids dans la balance de la misère et de l'injustice (p. 64).

La quête de l'eau : une symbolique centrale et une problématique transversale À Fonds-Rouge comme ailleurs, la question de l'eau, c'est la vie ou la mort, la salvation ou la perdition (Roumain, 2007, p. 38), un enjeu écologique central, collectif et permanent. Ici, la question de l'eau représente " l'élément qui peut remettre en marche la machine du système écologique [mais aussi] qui peut réconcilier les habitants » (Damus, 2010). Le titre Gouverneurs de la rosée, traduction du créole Mèt Lawouze, est éloquent : il évoque le maître de l'eau, celui qui gouverne l'eau, qui commande à l'eau et à qui l'eau obéit. Littéralement, Annaïse voit en Manuel, son amoureux, le maître de l'eau. La symbolique de l'eau, par la couleur bleue, est très présente dans le récit. Le prénom Annaïse, le diminutif de Anne, est associé au bleu ; et Manuel, en proposant à Annaïse de peindre la case qu'il leur construira en bleu, parce que Ça fait propre le bleu (p. 65), est encore dans la symbolique de l'eau, qui lave (le sang), nettoie et dissout les misères sociales et environnementales.

\title{
L'identité écologique de Manuel
}

\author{
Je suis ça : cette terre-là, et je l'ai dans le sang. Regarde ma couleur : on dirait que la \\ terre a déteint sur moi... \\ (Roumain, 2007, p. 50)
}


Nous sommes ce pays et il n'est rien sans nous, rien du tout. Qui est-ce qui plante, qui est-ce qui arrose, qui est-ce qui récolte?

(Roumain, 2007, p. 51) l'une concerne la réconciliation, l'eau et la vie: la réconciliation pour que la vie recommence, pour que le jour se lève sur la rosée, et l'autre concerne une invitation à la joie, au vivre ensemble, au travailler ensemble, ou en créole, au konbit : Et chantez mon deuil, chantez mon deuil avec un chant de coumbite (Roumain, 2007, p. 121). Ces deux phrases résonnent comme le testament socio-écologique à la fois de Manuel, le personnage du roman, et de Jacques Roumain, l'auteur du roman. Tout en se trouvant aux extrêmes de l'échelle sociale haïtienne, l'auteur et son personnage sont l'image l'un de l'autre, leurs parcours de vie se confondent, leurs pensées se font écho, ils parlent d'une même voix, leurs identités fusionnent. À son retour, après quinze années à Cuba, Manuel pourrait avoir trente-sept ans, l'âge qu'avait Roumain lorsqu'il a rédigé le roman, peu avant sa mort. Probablement, comme Roumain qui a beaucoup voyagé et a effectué de nombreux retours en Haïti, Manuel tente à son retour au pays de se ré-enraciner: Depuis ce jour d'aujourd'hui, je suis icitte pour le restant de ma vie. Toutes ces années passées, j'étais comme une souche arrachée... (p. 24). élément important de son identité. Il ne peut être un villageois comme les autres, car il porte en lui cet ailleurs qu'il a ramené dans ses bagages identitaires. Malgré sa critique sociale sévère envers Cuba, il garde de son expérience migratoire des savoirs-clés, et surtout une conscience critique qu'il met à profit dans la problématisation de la sécheresse. Damus (2010 b) voit dans la langue de Manuel ponctuée de mots espagnols, l'acculturation vécue à Cuba et qui revient exprimer une réalité vécue dans la même langue : " huelga ; bueno; compadre ; bayabondes; carajo ; parece; viejo ; hé, qué pas ? zafra ». Son vocabulaire exprime la réalité de travailleurs immigrés soumis aux caprices d'un «patron» qui les exploite. L'absence a décuplé sa conscience socio-environnementale, la distance lui a conféré de la perspective et le temps lui a conféré de l'acuité.

Manuel, le chercheur ethnologue. Tout en s'immergeant dans les rituels culturels de sa communauté, il observe les habitants, déchiffrant dans les rides de leurs visages l'écriture impeccable de la misère (Roumain, 2007, p. 28). Il pratique l'observation participante à partir de sa double perspective émique, de l'intérieur (identité), et étique, de l'extérieur (altérité). C'est un regard de chercheur ethnologue qu'il pose sur son village à son retour, il veut le comprendre et se met donc à son écoute. Tout comme Roumain l'ethnologue et sociologue, les propos de Manuel font référence à des savoirs ethnologiques, anthropologiques, sociologiques, culturels.

, le critique social. En une seule phrase, il opère une critique ironique des enjeux de race et de classe au sein de la société et de l'Église haïtiennes: Et sûrement qu'il y a des anges nègres pour faire le gros travail de la lessive des nuages ou balayer la pluie et mettre la propreté du soleil après l'orage pendant que les anges blancs chantent comme des rossignols toute la sainte journée ou bien soufflent dans de petites trompettes comme c'est marqué dans les images qu'on voit dans les églises (Roumain, 2007, p. 25).

Manuel, le scientifique. Homme de terrain, Il avait voulu se rendre compte: eh bien, il savait maintenant... (Roumain, 2007, p. 36), il mobilise ses connaissances et les malangas, c'est une plante qui vient de compagnie avec l'eau (p. 79) et continue à apprendre Un courant de fraîcheur circulait et c'était peut-être pourquoi les plantes volubiles et désordonnées

Éducation relative à l'environnement, Volume 12 | 2015 
poussaient si drues et serrées (p. 79). Il n'aborde pas la misère comme une calamité des dieux, mais comme une résultante de l'action humaine qu'il faut comprendre pour mieux la résoudre : Le bon Dieu n'a rien à voir là-dedans [...] Il y a les affaires du ciel et il y a les affaires de la terre, ça fait deux et ce n'est pas la même chose (p. 25). Il rejette les attributions causales externes, stables et incontrôlables (Lafortune, 1996, p. 40) pour adopter une posture pragmatique de recherche de son pouvoir d'action. Il refuse la soumission à une volonté divine: Mais la Providence, laisse-moi te dire, c'est le propre vouloir du nègre de ne pas accepter le malheur, de dompter chaque jour la mauvaise volonté de la terre (p. 33).

Manuel, l'éducateur. Dans ses conversations avec les villageois, l'intention éducative de Manuel est manifeste ; il veut montrer, décrire, expliquer, faire comprendre, faire réfléchir, notamment avec son ami Laurélien : Dans le retrait le plus inarticulé de son esprit accoutumé à la lenteur et à la patience, là où les idées de résignation et de soumission s'étaient formées avec une rigidité traditionnelle et fatale, un rideau de lumière commençait à se lever (p. 51). Sa mère perçoit la vérité de ses paroles : Tu as la langue habile et tu as voyagé dans les pays étrangers. Tu as appris des choses qui dépassent mon entendement (p. 34). Mais elle ressent le besoin de le mettre en garde: Tes paroles ressemblent à la vérité et la vérité est peut-être un péché (p. 26). Annaïse admire la profondeur de ses paroles : C'est comme pour l'eau, il faut fouiller profond dans tes paroles pour trouver leur sens [...] Jésus-Marie la Vierge, comme tu es savant, et toutes ces idées, elles viennent de ta tête? (p. 85).

Manuel, le personnage biblique. La personnalité de Manuel présente un caractère audelà de l'humain, quasi biblique, à la fois Adam, Moïse et Jésus. Il est à noter que le prénom Manuel est le diminutif d'Emmanuel ; celui-ci dérive de l'hébreu Immanu'el qui signifie « Dieu est avec nous ». Roumain, semble avoir voulu pousser son indigénisme jusqu'à proposer un récit cosmogonique universel dans la figure de simplicité et de sagesse d'un jeune paysan haïtien analphabète (Roumain, 2007, p. 87). En plus d'être Adam, l'incarnation de l'homme, Manuel conduit son village vers une sorte de Terre Promise à travers sa quête de l'eau; ici, c'est l'identité du Moïse dont il se revêt. Le dénouement final, sa mort dans les bras de sa mère, n'est pas sans rappeler le rituel sacrificiel chrétien, la mort de Jésus, le sauveur (Damus, 2010).

31 Manuel, l'amoureux. L'amour de Manuel se rapporte à trois éléments féminins: l'environnement-eau comme source de la vie, sa mère comme source de sa vie, et Annaïse comme source de son prolongement de vie (elle porte son enfant). Entre ses trois amours, il réalise pleinement son identité d'homme : le citoyen, le fils et le maripère. Son rapport à l'environnement est empreint d'un principe féminin et de sensualité : Si l'on est d'un pays, si l'on y est né, comme qui dirait natif-natal [...] c'est une présence, dans le cœeur, ineffaçable, comme une fille qu'on aime: on connait la source de son regard, le fruit de sa bouche, les collines de ses seins... (p. 16). Son amour a le visage de l'authenticité et de la confiance auxquelles il aspire (p. 60).

Manuel, l'agent de transformation sociale critique, éthique, politique et praxique. L'activisme de Manuel est politique au sens étymologique du terme, du grec « polis » ou cité ; tout ce qui concerne les affaires de la cité est d'ordre politique (Sauvé, 2011). Son engagement répond aux éléments relevés par Sauvé (2009, p. 159) «l'engagement de la plupart de ces acteurs s'enracine d'abord dans le local, comme lieu d'authenticité, de réappropriation créatrice [...] d'innovation sociale et d'action. Le local se vit dans la solidarité de proximité: vivre ici, ensemble, entre nous». Ce qu'il vise, c'est la transformation sociale du village, d'une pensée statique, résignée et théocratique en 
une pensée dynamique, critique et démocratique d'engagement civique, d'action collective. On ne peut dissocier les dimensions politique, critique, éthique et praxique de l'engagement puisque celui-ci «se fonde le plus souvent sur un système de valeurs caractérisé par un souci de l'autre, du collectif, par une préoccupation pour le respect et la promotion de l'intégrité de l'autre [...] et pour le partage » (Sauvé, 2009, p. 159).

Manuel, le mystérieux. Qui est véritablement cet homme? Le Manuel doux et amoureux semble cacher un homme volontaire, en colère et qui peut être violent, en référence à son expérience cubaine. Un halo de mystère demeure : quelle était sa vie à Cuba? Il avait promis de revenir bien vite, pourquoi ne revient-il que quinze années plus tard ? Pourquoi cette obsession de sauver le village ? Avait-il une faute à réparer? Que s'est-il passé avec les autorités cubaines? Comment s'est-il retrouvé en prison? Avait-il la mort d'un homme sur la conscience? Manuel d'un bond saisit le revolver, brisa le poignet du garde. Ils roulèrent sur le sol. L'homme voulut appeler au secours, Manuel d'un coup de crosse lui cassa les dents et frappa à coups redoublés jusqu'à enfoncer son arme dans le mou (p. 30). Sa vie de migrant à Cuba demeure un mystère et la violence de cet ailleurs avant le poursuit dans l'ici et maintenant de son village.

\section{Pertinence du roman « Gouverneurs de la rosée » dans le contexte socio-environnemental d'Haïti}

Nous avons beau ne pas te voir, et savoir désormais que tu es entré un peu plus intimement dans la sainte chimie du sol natal tu demeures notre leçon.

(Camille, 2007, cité par Fombrun, 2007)

Le narrateur du récit, qui se fait la conscience critique de Manuel, témoigne des actions inconséquentes des villageois et de leurs conséquences environnementales, la chaîne des actions anthropiques et de leurs conséquences écologiques. Mais, dans la réalité, comment reprocher à des paysans dont la vie dépend exclusivement de l'économie agro-sylvo-pastorale de déboiser leurs champs pour cultiver, exploiter le bois ou obtenir des lieux de pâturage ? La déforestation est paradoxalement une nécessité et un problème, et toutes les sociétés y ont procédé à divers degrés depuis l'époque médiévale pour transformer des surfaces forestières en surfaces cultivables ou pour obtenir du bois pour l'énergie et la construction (Damus, 2010). Ici, il faut noter le caractère à la fois novateur et visionnaire du discours écologique de Jacques Roumain qui fut élaboré dans les années quarante, c'est-à-dire, près de vingt ans avant «Le printemps silencieux » de Rachel Carson, texte qui marque l'émergence de la pensée écologiste.

L'avenir donnera raison à Jacques Roumain : « De 1940 à 2002, la couverture forestière d'Haïti est passée de $40 \%$ à $1 \%$ » (Michel, 2002, cité par Damus, 2010). Et, en effet, la fabrication du charbon de bois y est devenue l'une des causes principales de la déforestation (Louis, 2008). Citant Haïti Press Network (2004), Louis précise : « En 2004, des spécialistes ont fixé à quinze ans l'échéance pour la désertification totale d'Haïti [...] le taux de reboisement est quatre fois inférieur au taux du déboisement. En d'autres termes, pour quatre arbres abattus, les Haïtiens en plantent seulement un. Quatre millions de tonnes d'arbres sont abattus chaque année en Haïti à des fins énergétiques » (Louis, 2008, p. 193). Mais, pourquoi les Haïtiens utilisent-ils le bois comme source principale d'énergie? «En décembre 2007, il [le sac de charbon de bois] se vendait à 650 gourdes ou $17,10 \$$. [...] s'il trouve suffisamment de bois, il peut 
généralement produire en deux semaines entre quatre et six sacs de charbon, environ 130 sacs en un an, d'où un revenu théorique de quelque 2200 dollars, plus de dix fois le revenu annuel per capita d'Haïti " (Louis, 2008, p. 206). L'auteur estime que la cause fondamentale du déboisement en Haïti et de l'état de profonde détérioration de l'environnement qui en résulte réside dans les inégalités sociales: l'oligarchie dominante représente $4 \%$ de la population et possède $66 \%$ des ressources; la classe moyenne ne représente que $16 \%$ de la population et dispose de $14 \%$ des ressources économiques, culturelles et éducatives ; les classes dominées ou pauvres représentent $70 \%$ de la population et ne disposent que de $20 \%$ des ressources nationales ; $10 \%$ de la population ne dispose pas de ressources et ne vit que d'expédients ; la marginalisation ou l'exclusion de catégories sociales spécifiques est une des causes de la détérioration de l'environnement ; $80 \%$ de la population rurale vit dans la pauvreté ; en 2001, $56 \%$ de la population haïtienne vivait sous le seuil de la pauvreté extrême.

Même la solution du reboisement pose problème pour les petits agriculteurs : « Le projet de reboisement constitue un désavantage pour nous, [...] Ce sont des arbres qui ont besoin de beaucoup d'eau pour grandir. Ainsi, ils rendent nos terres cultivables plus arides. " (Propos d'un petit agriculteur cité par Louis, 2008, p. 199). De même, les solutions de rechange qui passent par le gaz de pétrole liquéfié (GPL) ou les réchauds améliorés sont circonscrites aux villes, sont inaccessibles pour la majorité de la population et sont aussi nuisibles à l'environnement en raison de leur production de gaz à effet de serre (Louis, 2008). La lutte contre la dégradation de l'environnement doit donc aller de pair avec la lutte contre les inégalités sociales en reconnaissant que «les problèmes de l'environnement en Haïti ont des déterminants sociaux, historiques et économiques qu'on ne peut négliger dans la lutte pour le reboisement» (Louis, 2008, p. 206). Selon Gantheret (2010), « En raison de la déforestation, le problème de l'eau est permanent à Haïti : durant la saison des pluies, l'eau est dévastatrice puis, durant la saison sèche, elle manque cruellement $»$.

\title{
Pour une éducation à l'environnement dans le contexte socio-environnemental d'Haïti
}

\author{
Il avait toujours regretté, Manuel, de ne pas savoir les écritures. Mais lorsque \\ l'existence, grâce à l'arrosage, sera devenue meilleure, on demandera au Magistrat \\ Communal du bourg d'installer une école à Fonds-Rouge. Il proposerait aux \\ habitants de bâtir de bonne volonté une case pour l'abriter. C'est nécessaire \\ l'instruction, ça aide à comprendre la vie. Témoin, ce compagnero à Cuba qui lui \\ parlait politique, au temps de la grève. Il en savait des choses, el hijo de... su madre, \\ et les situations les plus embrouillées, il te les démêlait que c'était une merveille : tu \\ voyais devant toi chaque question alignée sur le fil de son raisonnement comme du \\ linge rincé accroché à sécher au soleil; il t'expliquait l'affaire si claire que tu \\ pouvais la saisir comme un morceau de pain avec la main [...] Et si l'habitant allait à \\ l'école, certain qu'on ne pourrait plus si facilement le tromper, l'abuser et le traiter \\ en bourrique. \\ (Roumain, 2007, p. 112).
}

Dans cette citation, la réflexion de Manuel est en soi une ode à l'éducation et à l'école qui les associe à la lumière (soleil), l'ordre (démêlait), la precision (le fil de son raisonnement), la netteté (linge rincé), la limpidité (si clair), l'appropriation (saisir), la nourriture (le pain), les sens (la main). Pour ce qui est de l'éducation à l'environnement, nous nous référons aux éléments suivants : la dimension écologique 
ou l'éducation relative à l'environnement comme étant « toute forme d'éducation ayant trait au rapport à l'environnement, soit à l'ensemble des réalités socio-écologiques avec lesquelles nous interagissons..." (Sauvé, 2011, p.9); la dimension politique ou l'éducation relative à l'environnement comme étant «ce qui se passe quand les citoyens se réunissent pour discuter, débattre et décider les mesures politiques qui conditionnent leur existence en tant qu'habitants d'une ville ou d'un village » (Heller, 2002, citée par Sauvé, 2011, p. 9). Que ce soit dans un contexte formel, non formel ou informel, on peut dire qu'il y a éducation lorsqu'il y a une intention, explicite ou implicite, de partager des savoirs de tous ordres visant à comprendre hier, à coconstruire le vivre-ensemble d'aujourd'hui et à penser le devenir-ensemble de demain.

Pour mettre en évidence la dimension sociopolitique centrale dans l'activisme de l'auteur Jacques Roumain et dans l'engagement communautaire du personnage Manuel, nous nous inspirons du cadre de référence de Sauvé (2011) autour de la dimension politique de l'éducation relative à l'environnement. L'auteure retient deux conditions institutionnelles à mettre en place et quatre intentions éducatives à développer. Les deux conditions institutionnelles sont : 1) les politiques publiques comme nécessité et enjeux d'un support formel ; 2) une éducation au politique vers une écocitoyenneté. Les quatre apprentissages ou intentions éducatives sont : 1) apprendre ensemble, au cœur de l'action sociale ; 2) construire collectivement un savoir critique ; 3) s'inscrire dans une démarche éthique ; 4) valoriser l'engagement citoyen.

Le roman Gouverneurs de la rosée présente de manière quasi explicite une proposition d'éducation à l'environnement et l'identité écologique selon quatre dimensions qui font écho aux intentions éducatives de Sauvé (2011). Ce sont: la dimension politique (l'apprentissage sur/dans/par/pour le collectif); la dimension éthique (les valeurs partagées et leur mise en débat dialogique); la dimension critique (la déconstruction des réalités sociales oppressives et la dénonciation des rapports de pouvoir inéquitables); et la dimension praxique (la réflexion sur/dans/par/pour l'action engagée en vue de la transformation des réalités sociales oppressives).

La dimension politique suppose d'apprendre ensemble à apporter des solutions aux problèmes collectifs auxquels fait face le village. Dès son retour de l'étranger, Manuel s'attèle littéralement à une "lecture du monde ", de son monde qu'il observe, analyse et essaie de comprendre, et sur lequel il formule des hypothèses, les vérifie et tire des conclusions en vue de la planification de ses actions. Il note tout de suite les obstacles et entraves au vivre-ensemble et n'a de cesse d'amener les personnes à une réflexion. Cependant, c'est dans le partage du fruit de ses apprentissages avec sa communauté que ceux-ci prennent toute leur valeur de savoirs et tout leur sens pour faire face aux défis collectifs.

41 La dimension éthique suppose la clarification des valeurs partagées, la reconnaissance mutuelle des valeurs affirmées et la mise en débat continue de ces valeurs. Comme le soutient Sauvé (2011), qui dit éducation politique dit aussi entrée dans l'univers complexe des valeurs et leur clarification, mais sans espérer un consensus éthique qu'elle juge bien improbable. Tout en respectant les valeurs traditionnelles des gens du village, Manuel infuse des valeurs nouvelles de dialogue, de solidarité, de justice sociale, d'inclusion, d'amour, de travail et de respect de l'environnement. Il dénonce une certaine morale religieuse axée sur l'autorité théocratique et propose une éthique de type humaniste, démocratique et écologique. Là aussi, Manuel forge son identité, puisque la délibération éthique suppose « un processus d'introspection, un moyen de 
découverte de soi à travers son identification avec un ensemble plus vaste : le domaine partagé de notre humanité » (Thomashow, 1995, cité par Sauvé, 2011, p. 16).

La dimension critique suppose la déconstruction des réalités socio-environnementales oppressives. Elle suppose également la reconstruction des rapports de pouvoir et de savoir qui sont la cause de ces oppressions. Manuel remet en question la mentalité traditionnelle de soumission aux dieux africains et occidental, d'impuissance apprise, d'exploitation inconsidérée de l'environnement en vue de rompre les dynamiques d'aliénation et de développer des dynamiques de transformation de la collectivité et d'émancipation des personnes. De plus, son expérience de coupeur de canne à l'étranger lui a bien fait comprendre la nécessité de résister aux exactions des élites sociales aux dépens des travailleurs, et c'est avec un argumentaire bien soutenu qu'il critique les forces sociales oppressives. Manuel aura effectué « le repérage et l'analyse des dysfonctions socio-écologiques et l'analyse des relations de pouvoir qui ont causé ou entretiennent ces problèmes d'aliénation sociale ou de détérioration du milieu de vie - ces derniers étant souvent reliés entre eux » (Sauvé et Orellana, 2008, citées par Sauvé, 2011, p. 14).

La dimension praxique suppose le sentiment de responsabilité face aux divers défis qui se présentent à la communauté, le fait de se sentir concerné par eux et la volonté de participer aux délibérations de manière démocratique et aux actions collectives. Manuel n'attend pas, n'espère pas non plus un secours qui viendrait de l'extérieur du village fut-ce-t-il gouvernemental, international ou divin. Dans le sens du concept freirien de "praxis » (Freire, 1974), Manuel invite sa communauté à s'impliquer dans un processus de réflexion-action au cours duquel ils «prennent un recul critique par rapport à leur environnement familier, pour le percevoir sous un angle à la fois différent et critique. Ils sont encouragés à "revivre l'ordinaire de manière extraordinaire"... » (Mayo, 2007). Tout au pays, avant même d'agir ou de reconstruire, les Haïtiens doivent d'abord rêver et penser la nouvelle Haïti (Bourjolly, 2010).

\section{Conclusion}

Dans cet article, nous avons voulu mettre en évidence les dimensions identitaire et écologique du roman Gouverneurs de la rosée de l'écrivain haïtien Jacques Roumain en nous attardant au cheminement du personnage principal, Manuel, dans le contexte socio-environnemental d'Haïti des années quarante. Or, loin d'être un texte dépassé, ce roman se révèle d'actualité, et même plus significatif aujourd'hui compte tenu des défis socio-environnementaux criants auxquels fait face Haïti. Aussi il se révèle tout à fait pertinent dans le questionnement portant sur le sens du lieu et sur le rapport à l'environnement des Haïtiens (Prévil et Thésée, 2012).

Manuel pose sur son village un regard neuf, éclairé et amplifié par sa perspective du voyageur qui écrit son cahier du retour au pays natal à la manière d'Aimé Césaire. Il va y déployer tout son vouloir-agir, son pouvoir-agir et son savoir-agir en vue de dénouer l'impasse socio-environnementale de l'eau dans laquelle se trouve plongé Fonds-Rouge. Par les dimensions politique, éthique, critique et praxique de son action, c'est bien une éducation à l'environnement et, plus largement, une éducation à l'identité écologique qui résultent des actions de Manuel. Derrière son personnage, l'auteur Jacques Roumain, à la veille de sa mort, se pose en un véritable éducateur critique. Il a élevé son activisme sociopolitique au rang de l'art et s'est servi de son art littéraire 
pour en faire un programme d'éducation, une éducation à l'environnement, à la paix, à la justice sociale, à la solidarité, à la démocratie et à l'identité écologique. Son roman " Gouverneurs de la rosée » est un véritable testament écologique offert à son pays et au monde.

Le roman "Gouverneurs de la rosée» a une précieuse valeur éducative, environnementale, sociale et politique. En conclusion de cet article, nous faisons le vœu qu'il soit mis à profit dans la conception d'un projet éducatif, d'un programme scolaire, d'un programme de formation à l'enseignement ou d'un modèle d'enseignementapprentissage dans les domaines de l'éducation à l'environnement, de l'éducation pour la démocratie et de l'identité écologique en Haïti même et ailleurs dans le monde. Aussi, il serait souhaitable de révéler l'éducation relative à l'environnement dans d'autres romans, alliant ainsi l'ERE à la littérature.

\section{BIBLIOGRAPHIE}

Abramson, L. Seligman, M. et Teasdale, J.D. (1978). Learned helplessness in humans : critique and reformulation. J. Abnormal Psychology, 87, 49-74.

Bourjolly, J.-M. (2010). Haïti : quelle reconstruction ? Dans Refonder Haïti ? (Sous la dir. De P. Buteau, R. Saint-Éloi et L. Trouillot). Montréal : Mémoire d'encrier, p. 25-45.

Carr, P.R., Pluim, G. W. J. et Thésée, G. (2014). O contexto da vulnerabilidade ambiental enquanto um produto da colonização e da democracia liberal : o entendimento e a educação sobre o terremoto no Haiti. Em Aberto, 27 (91), 135-156

Cottereau, D. (2012). Habiter par corps. Éducation relative à l'environnement : Regards Recherches - Réflexions, vol. 10, p. 17-34.

Damus, O. (2010 a). Rapports entre l'homme et l'environnement dans le récit de Jacques Roumain (1907-1944) : Gouverneurs de la rosée (Roman haïtien). Présentation dans le cadre de la Conférence de l'Association de littérature africaine (ALA) ; Tucson, Arizona, 10-14 mars 2010.

Damus, O. (2010 b). Le phénomène migratoire dans le récit de Jacques Roumain : Gouverneurs de la rosée. Consulté dans le site Potomitan de promotion des cultures et des langues créoles : www.potomitan.info/ayiti/gouverneurs_rosee2.php.

Fombrun, C. (2007). Hommage à Jacques Roumain. Conférence dans le cadre des activités de commémoration du 100e anniversaire de naissance de Jacques Roumain. Site Potomitan de promotion des cultures et des langues créoles.

Freire, P. (1974). Pédagogie des opprimés. Paris : François-Maspero.

Ganthret, L. (2010). Capitaines de l'espérance/Haïti. Émission diffusée sur TV5 Monde les 24, 25 et 26 mars 2012.

GRAHN (2010). GRAHN-Monde : Groupe de réflexion et d'action pour une Haïti nouvelle. Consulté dans le site : www.haiti-grahn.net/public/ 
Hoffmann, L.-F. (2003). Introduction du coordinateur. Dans Jacques Roumain, Æuvres complètes. Paris : Archivos, p. XXXI-XLVII.

Hoffmann, L.-F. (2003). Biographie de Jacques Roumain. Dans Jacques Roumain, đeuvres complètes. Paris : Archivos. Consulté dans le site : www.lehman.cuny.edu/ile.en.ile/paroles/ roumain_bio.html

Jeanty, G. J. (2012). Bayahonde, une source de revenu mal exploitée. Dans le quotidien haïtien Le Nouvelliste, 6 décembre 2012.

Laferrière, D. (2007). Texte de quatrième de couverture. Dans Gouverneurs de la rosée. (Jacques Roumain : Auteur). Montréal : Mémoire d'encrier.

Lafortune, L., Saint-Pierre, L. (1996). L'affectivité et la métacognition dans la classe. Montréal : Logiques.

Louis, L. (2008). Haïti : combattre le déboisement ou les inégalités sociales ? Alternatives Sud. Déforestation. Causes, acteurs et enjeux, 15 (3), 193-209. Paris : Éditions Sylepse.

Mayo, P. (2007). 10e Anniversaire de la mort de Paulo Freire. Éducation des adultes et Développement, 69. Consulté dans le site : www.iiz-dvv.de/index.php ?article_id =284\&clang =2

Planète Avenir (2004). Informations sur les technologies traditionnelles en Haïti. www.haiticulture.ch/Env_techno_traditions.html

Prévil, C. et Thésée, G. (2012). Développer le sens du lieu dans l'éducation et la formation en Haïti : une perspective critique. Dossier : Présences haïtiennes. Dialogues et cultures, 58, 119-128. Roumain, J. (2007, 1er ed.1944). Gouverneurs de la rosée. Montréal : Éditions Mémoire d'encrier Roumanes, J.-B. (1993). La notion d'identité ; essai de définitions : de l'identité logique à l'identité de la personne. Dans La question de l'identité. Qui suis-je ? Qui est l'autre ? (Dir. C. Gohier et M. Schleifer). (p. 227-257). Montréal : Les Éditions Logiques.

Sauvé, L. (2011). La dimension politique de l'éducation relative à l'environnement - Un certain vertige. (Texte éditorial). Éducation relative à l'environnement : Regards - Recherches Réflexions, 9, 7-21.

Sauvé, L. (2009). Le rapport entre éthique et politique : un enjeu pour l'éducation relative à l'environnement. Éducation relative à l'environnement : Regards - Recherches - Réflexions, 8, 147-162.

Sauvé, L. (2001). Éducation et environnement à l'école secondaire. Outremont (Québec) : Les Éditions Logiques.

Thésée, G. (2003). Le rapport au savoir scientifique en contexte d'acculturation : Application à l'étude de l'expérience scolaire en sciences d'élèves du secondaire d'origine haïtienne. Thèse de doctorat. Montréal : Université du Québec à Montréal.

\section{RÉSUMÉS}

Le roman «Gouverneurs de la rosée » de l'écrivain haïtien Jacques Roumain présente un caractère identitaire dont les dimensions socio-politique et écologique sont proéminentes. Manuel, le personnage central du roman, fait écho au militantisme socio-politique et scientifique de l'auteur. Nous menons une analyse de l'identité écologique du personnage principal du roman, Manuel, aux plans individuel, social et écologique. Le discours de l'auteur qui rend hommage à la 
poésie du langage vernaculaire haïtien est mis en valeur. En synthèse, nous esquissons ce que nous pensons être le message central du roman : une proposition d'éducation à l'environnement et d'identité écologique dans le contexte socio-environnemental d'Haïti.

Haitian writer Jacques Roumain's book Gouverneurs de la rosée presents a protoype of a character and identity, of which socio- political and ecological dimensions are prominant. The qualities of the primary character, Manuel, are reflected and rendered more transparent through the well-known socio-political and scientific activism of the author. We explore the ecological identity of the main character in the book while underscoring the literary value of the poetic and Haitian vernacular narrative presented by the author. In sum, we trace what we believe to be the central message of this important literary work: a proposal for environmental education and ecological identity within a socio-environmental Haitian context.

\section{INDEX}

Mots-clés : environnement, identité, identité écologique, politique, Jacques Roumain Index géographique : Haïti

Keywords : environment, identity, ecological identity, politics

\section{AUTEURS}

\section{GINA THÉSÉE}

Professeure au Département de didactique de l'Université du Québec à Montréal. Ses intérêts de recherche ont trait à l'éducation en contextes de vulnérabilités multiples dues aux discriminations et oppressions liées au genre, à la race, la culture, l'ethnicité, le colonialisme. À partir d'une perspective sociocritique et transdisciplinaire, elle intègre la pédagogie, la didactique, l'épistémologie et l'écologie. Son cadre théorique conceptuel prend racines dans le féminisme, l'antiracisme, l'environnementalisme, l'anticolonialisme, l'indigénisme, le multi/ inter/trans/culturalisme.

\section{PAUL R. CARR}

Professeur au Département des sciences de l'éducation de l'Université du Québec en Outaouais. Ses champs de recherche comprennent la sociologie politique, l'éducation interculturelle, la démocratie, l'alphabétisation des médias, les études de la paix et la justice socioenvironnementale. Il dirige le projet de recherche CRSH Démocratie, alphabétisation politique et éducation transformatoire. Ses nombreuses publications comprennent des articles, chapitres, livres édités et livres dont le Does Your Vote Count? Critical Pedagogy and Democracy. 